\title{
Tetrandrine reverses epithelial-mesenchymal transition in bladder cancer by downregulating Gli-1
}

\author{
YONGJIAN ZHANG ${ }^{1 *}$, WEI LIU ${ }^{2 *}$, WENBO HE $^{4 *}$, YUANYUAN ZHANG ${ }^{5}$, \\ XIULING DENG ${ }^{4}$, YANMIN MA ${ }^{3}, \mathrm{JIN} \mathrm{ZENG}^{2}$ and $\mathrm{BO} \mathrm{KOU}^{1}$ \\ Departments of ${ }^{1}$ Cadiovascular Sugery, ${ }^{2}$ Urology and ${ }^{3}$ Reproductive Medicine, \\ First Affiliated Hospital of Medical School, ${ }^{4}$ Medical School, \\ Xi'an Jiaotong University; ${ }^{5}$ Department of Burn and Plastic Surgery, \\ Xi'an Central Hospital, Xi'an, Shaanxi 710061, P.R. China
}

Received December 24, 2015; Accepted February 4, 2016

DOI: $10.3892 /$ ijo.2016.3415

\begin{abstract}
Hedgehog (Hh) signaling pathway is considered to play a crucial role in vertebrate development and carcinogenesis. Additionally, epithelial-mesenchymal transition (EMT) is a cellular process during which epithelial cells become mesenchymal-appearing cells, facilitating cancer metastasis and invasion. Accumulating evidence has indicated that the Hh signaling pathway could potentiate the epithelial-mesenchymal transition (EMT). In the present study, we demonstrated that tetrandrine, a bisbenzylisoquinoline alkaloid isolated from Stephaniae, exerts its anti-metastatic ability in bladder cancer cells by regulating GLI family zinc finger 1 (Gli-1), a key factor of Hedgehog signaling pathway. In our study, we confirmed that tetrandrine could impede migration and invasion in bladder cancer 5637 and T24 cells. Additionally, tetrandrine reverses EMT by increasing the expression of E-cadherin and reducing the $\mathrm{N}$-cadherin, vimentin and Slug expression in a dose-dependent manner. Interestingly, tetrandrine also decreases mobility and reduces the expression of Gli-1 in bladder cancer cells. Moreover, we verified that tetrandrine inhibits metastasis and induces mesenchymal-epithelial transition (MET) of bladder cancer through downregulation of Gli-1, which could be partially reversed by Gli-1 overexpression. In conclusion, our findings show that tetrandrine inhibits migration and invasion, and reverses EMT of bladder cancer cells through negatively regulating Gli-1. It indicates that Gli-1
\end{abstract}

Correspondence to: Dr Bo Kou, Department of Cadiovascular Sugery, First Affiliated Hospital of Medical School, Xi'an Jiaotong University, 277 Yanta West Road, Xi'an, Shaanxi 710061, P.R China

E-mail: 821957325@qq.com

*Contributed equally

Key words: tetrandrine, bladder cancer, migration, invasion, epithelialmesenchymal transition, Gli-1 may be a potential therapeutic target of tetrandrine against bladder cancer.

\section{Introduction}

Bladder cancer is the most common malignant tumor of the urinary system worldwide, accounting for 74,000 new cases and 16,000 deaths annually in 2015 (1). Despite intense efforts toward improving the therapeutic success in treating bladder cancer, $\sim 10 \%$ non-muscle-invasive bladder cancer ultimately progressed to muscle-invasive bladder cancer (2). Tendency to invade and transfer is believed to be a major cause of treatment failure in bladder cancer.

Epithelial-to-mesenchymal transition (EMT) is of great necessity for the initiation of the metastatic cascade. The process confers migratory, invasive and stem-like properties upon cells (3), and epithelial polarized cells ultimately turn into motile mesenchymal-appearing cells (4). In epithelial cells during EMT, cellular phenotype has a series of changes, including the loss of cell-cell contacts, cell polarity and epithelial markers, particularly E-cadherin. Moreover, cells acquire mesenchymal-appearing properties. Additionally, cellular cytoskeleton change during EMT confers mesenchymal phenotypes, such as increased motility $(5,6)$. The induction of EMT can cause cancer cells to invade through the basement membrane and colonize into the distant solid organs. Hence, the EMT process is essential for the invasion-metastasis cascade of cancer.

The Hedgehog $(\mathrm{Hh})$ signaling pathway has been implicated to vertebrate development, cell differentiation and carcinogenesis (7). It was reported that the $\mathrm{Hh}$ signaling pathway is aberrantly expressed in various cancers, including breast cancer (8), prostate cancer (9) and lung cancer (10). Moreover, accumulating evidence shows that Gli-1, a crucial factor in the Hedgehog (Hh) signaling pathway, could increase the expression of N-cadherin and Snail, while decrease the expression of E-cadherin (11). Furthermore, Hedgehog signaling pathway enhanced invasive and metastatic potential of hepatocellular carcinoma (12). These results suggest that there may be a close association in the occurrence of EMT and metastasis with Hedgehog signaling pathway. 
Tetrandrine (TET), isolated from traditional Chinese medicine Stephaniae, is widely used for a broad spectrum of pharmacological events, including antihypertension, antisilicosis and antiarrhythmia. Accumulating evidence has shown the strong anticancer effect of tetrandrine. For many tumors such as prostate cancer $(13,14)$, colon cancer $(15,16)$, gastric cancer (17), esophageal squamous carcinoma (18), tetrandrine exhibited beneficial impact on reversion of multidrug resistance $(18,19)$, apoptosis induction, cell cycle arrest, inhibition of angiogenesis, chemosensitization, inhibition of metastasis (20), involved in ROS/AKT pathway (21), Wnt/ $\beta$ signaling pathway (22), intrinsic apoptotic signaling pathway, and mitogen-activated protein kinase activation.

Li et al (23) showed that tetrandrine exerts anticancer effects against bladder cancer via growth inhibition and apoptosis induction. In spite of its anticancer potential, however, the anti-metastasis effect and the underlying mechanism of tetrandrine on bladder cancer has not yet been elucidated. In particular, there have been no studies showing whether tetrandrine inhibits the Hh signaling pathway to regulate the EMT in bladder cancer. The aim of the present study was to focus on the potential of tetrandrine as an inhibitor of the Hh pathway, which reverses EMTs and inhibits migration and invasion, in bladder cancer.

\section{Materials and methods}

Reagents and cell culture. Tetrandrine $\left(\mathrm{C}_{38} \mathrm{H}_{42} \mathrm{~N}_{2} \mathrm{O}_{6}\right)$ was purchased from Sigma-Aldrich (St. Louis, MO, USA) and dissolved with $0.1 \mathrm{~mol} / 1 \mathrm{HCl}$ at a concentration of $25 \mathrm{mg} / \mathrm{ml}$. Stock solutions were stored at $-20^{\circ} \mathrm{C}$ and diluted with medium in certain proportions immediately prior to use. Antibodies against E-cadherin, N-cadherin, vimentin, Slug, Gli-1, $\beta$-actin and peroxidase-conjugated secondary antibodies were obtained from Cell Signaling Technology, Inc. (Beverly, MA, USA). 3-(4,5-dimethylthiazol-2-yl)-2,5-diphenyltetrazolium bromide (MTT) were obtained from Sigma Chemical Co. (St. Louis, MO, USA). The enhanced chemiluminescence (ECL) detection system was obtained from Amersham Life Science, Inc. (Arlington Heights, IL, USA).

Human bladder cancer cell lines 5637, T24, 253, BV, J82 and UC3, and bladder epithelial SVHUC-1 cell were obtained from the American Type Culture Collection (Manassas, VA, USA). These cell lines were cultured in 1640/Dulbecco's modified Eagle's medium supplemented with $10 \%$ fetal bovine serum (Gibco, Grand Island, NY, USA) and $1 \%$ penicillinstreptomycin (Invitrogen, Carlsbad, CA, USA) at $37^{\circ} \mathrm{C}$ in a humidified atmosphere with $5 \% \mathrm{CO}_{2}$.

Cell proliferation assay. A modified MTT assay was used to detect cell proliferation viability. Briefly, 5637 and T24 cells were seeded at a density of $1 \times 10^{4}$ cells with $90 \%$ density in 96-well plates and treated with increasing doses of tetrandrine for $24 \mathrm{~h}$. Then, $20 \mu \mathrm{l} \mathrm{MTT}$ dye solution $(5.0 \mathrm{mg} / \mathrm{ml})$ was added to each well and incubated at $37^{\circ} \mathrm{C}$ for $4 \mathrm{~h}$. After incubation, the culture medium was discarded and the cells were lysed with dimethyl sulfoxide to dissolve the formazan crystals. The optical density (OD) of each well was detected at a wavelength of $490 \mathrm{~nm}$ using a 96-well microplate reader (Bio-Rad, Hercules, CA, USA). The percentage of cell growth inhibition resulting from tetrandrine was calculated as: [(OD490 control cells $^{-}$ OD $490_{\text {treated cells }}$ ) $/ O D 490_{\text {control cells }}$ ] x 100. The experiments were performed in triplicate.

Wound healing assay. Bladder cancer 5637 or T24 cells were seeded onto 6-well plates. When the cell density reached $>90 \%$, scratch wounds were made across the monolayer using the tip of a $200-\mu 1$ pipette. Then the wounded cultures were incubated in a serum-free medium with various concentrations of tetrandrine at different times, and five random fields (x100) were chosen from each scratch wound and visualized by microscopy to assess the ability of cell migration. The experiments were performed in triplicate.

Transwell migration assay. Transwell migration assays were performed using bladder cancer 5637 and T24 cells after treatment with tetrandrine. Cells (5637: $6 \times 10^{4}$ or T24: $3 \times 10^{4}$ ) with $200 \mu \mathrm{l}$ serum-free medium were plated onto the top chamber, and $800 \mu \mathrm{l}$ of medium supplemented with $10 \%$ fetal calf serum were added to the lower chamber. After incubation at $37^{\circ} \mathrm{C}$ for various times, cells adhering to the top chambers were wiped out with a cotton swab. The migratory cells on the lower surface of the filter were fixed with $4 \%$ paraformaldehyde and stained with $0.1 \%$ crystal violet (Beyotime, Shanghai, China). Cells that had migrated to the lower surface were counted in five randomly chosen visual fields under a microscope at x100 magnification. All of the data were obtained from three independent experiments.

Matrigel invasion assay. The impact of tetrandrine on the invasion of bladder cancer cells was determined by Matrigel invasion assay using a Millicell chamber (Millipore, Billerica, MA, USA). The membrane (polycarbonic membrane, $6.5 \mathrm{~mm}$ diameter, $8-\mu \mathrm{m}$ pore size) in the upper chamber was coated with $50 \mu$ l Matrigel (Matrigel: serum-free medium 1:5). After incubation at $37^{\circ} \mathrm{C}$ for $5 \mathrm{~h}$, the cells $\left(5637\right.$ : $12 \times 10^{4}$ or T24: $6 \times 10^{4}$ in $200 \mu \mathrm{l}$ medium of serum starvation, respectively) were treated by the same procedures as described above, similarly to the Transwell migration assay.

Quantitative real-time PCR assay. 5637 and T24 cells were cultured in the experimental conditions and their total RNA was extracted using TRIzol reagent (Invitrogen, Carlsbad, CA, USA) following the manufacturer's protocol. Then complementary DNA (cDNA) was synthesized using a PrimerScript RT reagent kit (Takara, Dalian, China). Additionally, the relative levels of target gene messenger RNA (mRNA) transcript were detected by quantitative real-time PCR assay (qRT-PCR) using the SYBR Green Master Mix. The sequences of primers for the PCR amplification were forward, 5'-AGCGTGAGCCTG AATCTGTG-3' and reverse, 5'-CAGCATGTACTGGGCTTT GAA-3' for Gli-1 (188 bp); forward, 5'-CGAGAGCTACACGT T CACGG-3' and reverse, 5'-GGGTGTCGAGGGAAAAAT AGG-3' for E-cadherin (119 bp); forward, 5'-TCAGGCGTCTG TAGAGGCTT-3' and reverse, 5'-ATGCACATCCTTCGATAA GACTG-3' for N-cadherin (94 bp); forward,5'-GACGCCATCA ACACCGAGTT-3' and reverse, 5'-CTTTGTCGTTGGTTAGC TGGT-3' for vimentin (238 bp); forward, 5'-CGAACTGGACA CACATACAGTG-3' and reverse, 5'-CTGAGGATCTCTGGT TGTGGT-3' for Slug (87 bp); forward,5'-CATGTACGTTGCTA 
A<smiles>[AsH]</smiles>

B

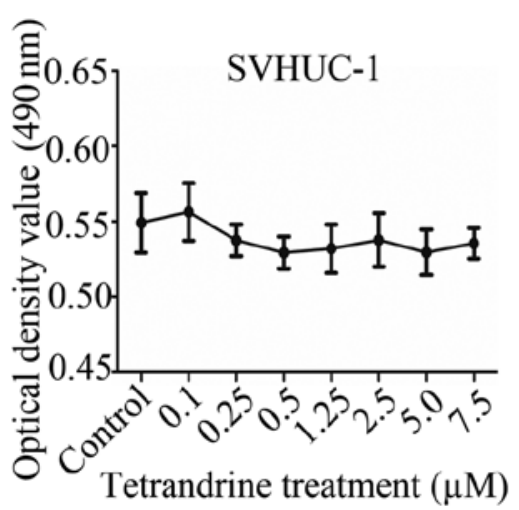

C

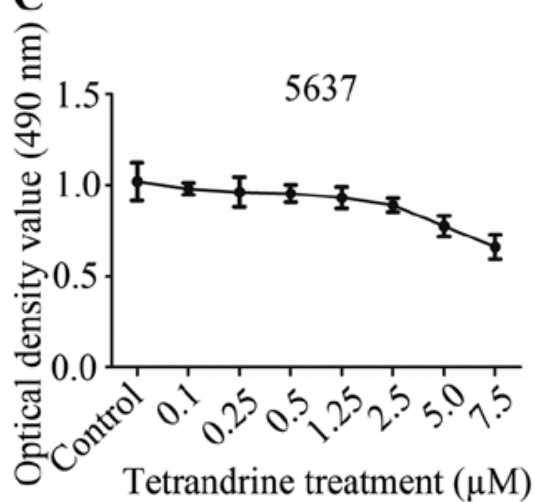

D

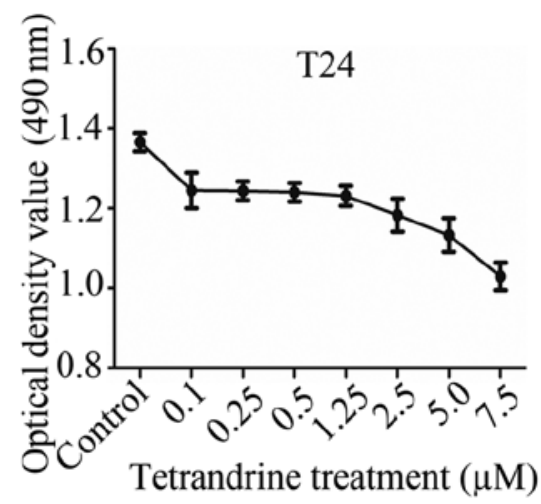

E

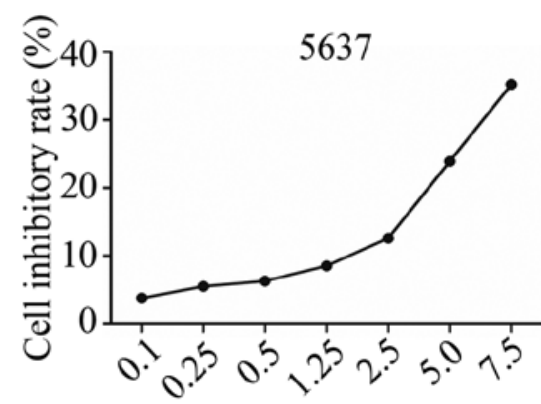

Tetrandrine treatment $(\mu \mathrm{M})$

F

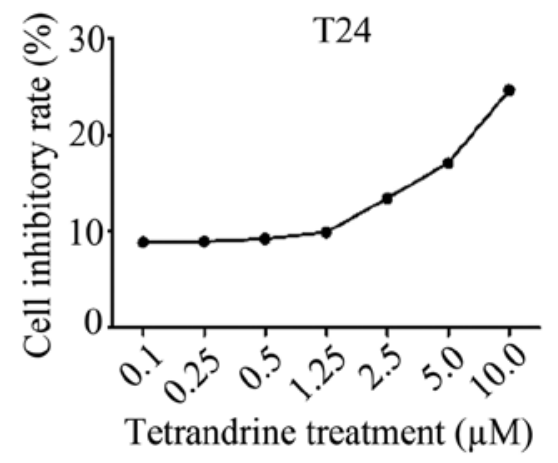

Figure 1. Tetrandrine inhibited the growth of bladder cancer cells. (A) Chemical structure of tetrandrine. (B) Cytotoxic effect of tetrandrine on normal bladder epithelial SVHUC-1 cells. The 5637 and T24 cells with $90 \%$ density were treated with various doses of tetrandrine for 24 h, the viability of these two bladder cancer cells was determined by MTT assays. The mean cell number of three independent experiments performed in triplicate is shown for 5637 (C and E) and T24 (D and F) cells.

TCCAGGC-3' and reverse, 5'-CTCCTTAATGTCACGCAC GAT-3' for $\beta$-actin (250 bp). All assays were performed in triplicate and were calculated on the basis of the $\Delta \Delta \mathrm{Ct}$ method. The $n$-fold change in mRNAs expression was determined according to the method of $2^{-\Delta \Delta C t}$.

Western blotting. Briefly, the bladder cancer cells were harvested $24 \mathrm{~h}$ after the tetrandrine treatment, and lysed on ice for $10 \mathrm{~min}$ with a lysis buffer $[10 \mathrm{mmol} / \mathrm{l}$ Tris- $\mathrm{HCl}(\mathrm{pH} 7.4)$, $150 \mathrm{mmol} / 1 \mathrm{NaCl}, 0.1 \%$ sodium dodecyl sulfate (SDS), $1 \mathrm{mmol} / \mathrm{l}$ ethylenediaminetetraacetic acid, $1 \mathrm{mmol} / \mathrm{l}$ ethylene glycol tetraacetic acid, $0.3 \mathrm{mmol} / 1$ phenylmethylsulfonyl fluoride, $0.2 \mathrm{mmol} / 1$ sodium orthovanadate, $1 \% \mathrm{NP}-40$, $10 \mathrm{mg} / \mathrm{ml}$ leupeptin and $10 \mathrm{mg} / \mathrm{ml}$ aprotinin]. After centrifugation, the clarified protein lysates $(\sim 30-60 \mu \mathrm{g})$ were separated by SDS-polyacrylamide gel $(10 \%)$ and transferred to polyvinylidene membranes (Millipore, Bedford, MA, USA). Immunoblotting was subsequently performed with the primary antibody against E-cadhrein, N-cadherin, vimentin, as well as Slug, and $\beta$-actin overnight at $4^{\circ} \mathrm{C}$. The membranes were then washed and incubated with horseradish peroxidase (HRP)-conjugated secondary antibody at room temperature $\left(25^{\circ} \mathrm{C}\right)$. Ultimately, the protein bands were visualized with ECL substrate and exposed to X-ray film.

Immunofluorescence microscopy. The immunofluorescence staining of F-actin was performed following the manufacturer's protocol. In brief, cells on slides were washed and fixed with 4\% paraformaldehyde for $15 \mathrm{~min}$. After being washed, cells were permeabilized with $0.1 \%$ Triton X-100 for $5 \mathrm{~min}$. Then cells were stained with $20 \mu \mathrm{l} \mathrm{F}$-actin for $30 \mathrm{~min}$ and DAPI dye for 5 min. Nikon (Tokyo, Japan) A1 confocal microscope was used for the observation of micrographs.

Plasmid transfection. Gli-1 cDNA was cloned into pcDNA3.1 vector. When bladder cancer 5637 or T24 cells achieved $70-80 \%$ confluency for plasmid transfection, the cells were transfected with X-treme Gene HP DNA transfection reagent (Roche, Germany) for $48 \mathrm{~h}$ according to the manufacturer's instructions, and harvested for the turnover experiments.

Statistical analysis. GraphPad Prism (vesion 5.0) software was used for all statistical analyses, and Student's t-test (two-sided) was used for comparisons involving only two groups. A value of $\mathrm{P}<0.05$ was considered statistically significant.

\section{Results}

The antiproliferative effect of tetrandrine in bladder cancer 5637 and T24 cells. Fig. 1A shows the chemical structure of tetrandrine with the molecular structural formula $\mathrm{C}_{38} \mathrm{H}_{42} \mathrm{~N}_{2} \mathrm{O}_{6}$ and a molecular weight of $622.74988 \mathrm{~g} / \mathrm{mol}$. The MTT assays showed that tetrandrine did not have a significant inhibitory effect on normal bladder epithelial SVHUC-1 cells (Fig. 1B), 
A
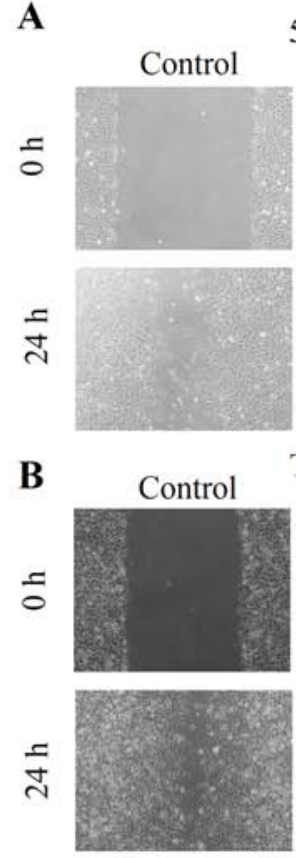

5637

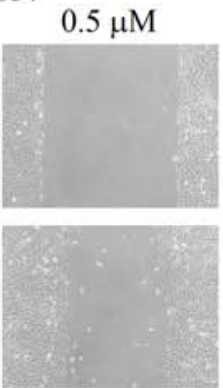

T24

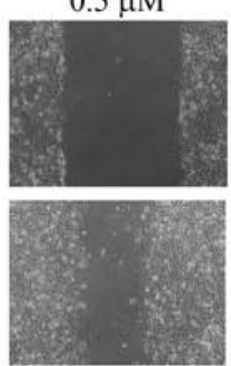

C

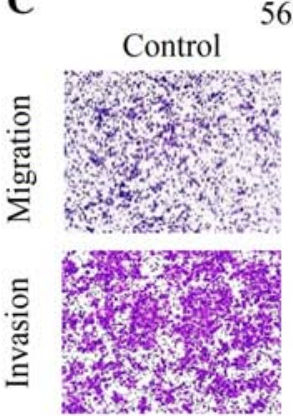

5637

$0.5 \mu \mathrm{M}$

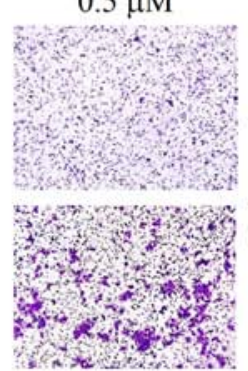

D Control

$\mathrm{T} 24$

$0.5 \mu \mathrm{M}$
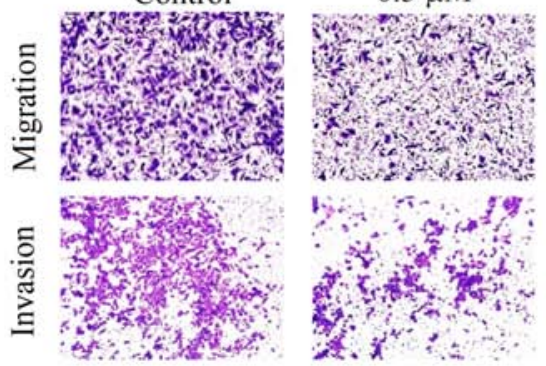

5637

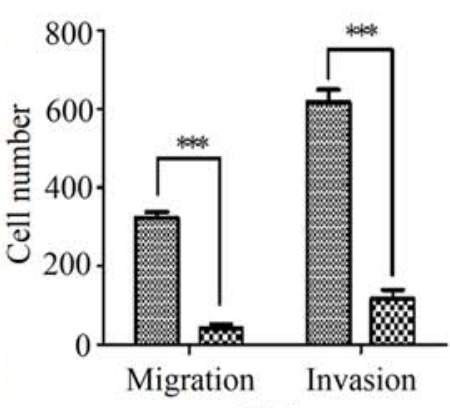

$\mathrm{T} 24$

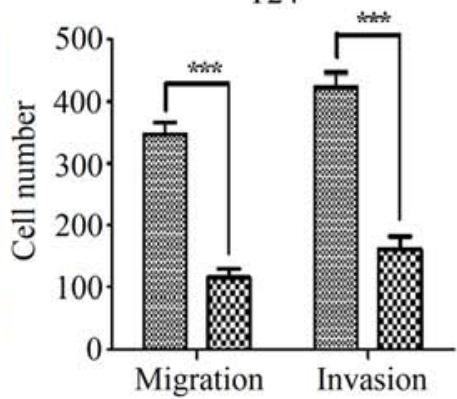

Control

$\mathbb{\mathrm { m }}$ Tet

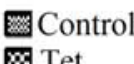

$\mathrm{MTet}$

Figure 2. Tetrandrine (Tet) represses the migration and invasion of bladder cancer 5637 and T24 cells. The width of scratches was measured with the treatment of tetrandrine, or not, at 0 and $24 \mathrm{~h}$ in bladder cancer 5636 (A) and T24 (B) cells. Additionally, using Transwell migration assay and Matrigel invasion assay, the number of migrated or invaded cells $(\mathrm{C}$ and $\mathrm{D})$ treated with tetrandrine per chamber was counted and compared with that in control group from three independent experiments $(\mathrm{P}<0.05)$.

A

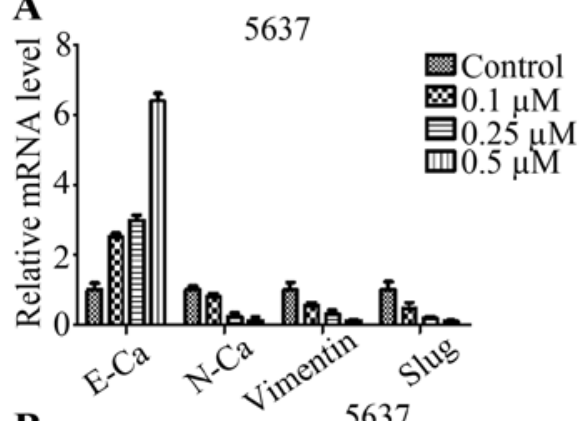

B

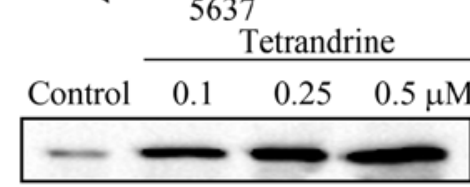

$\mathrm{N}-\mathrm{Ca}$

Vimentin

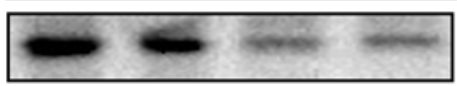

Slug

$\beta$-actin
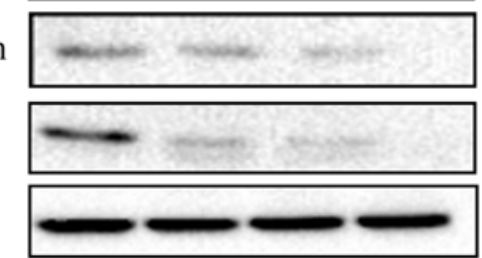

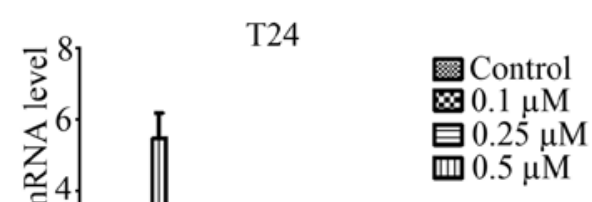

C
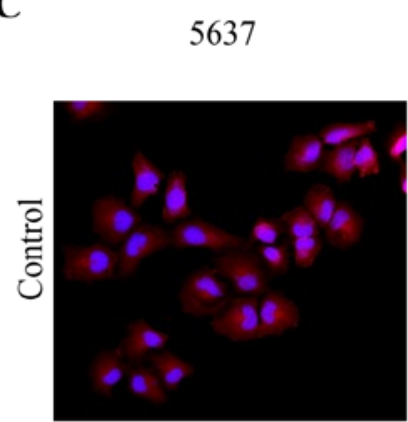

$\mathrm{T} 24$ Tetrandrine

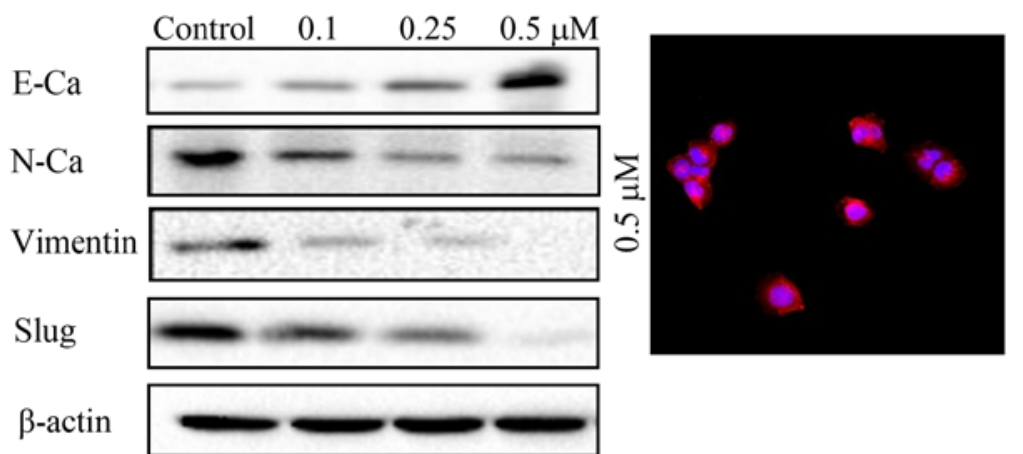

Figure 3. Tetrandrine blocks epithelial-mesenchymal transition in bladder cancer cells. (A) Quantitive real-time PCR was performed to examine the expression of E-cadherin, N-cadherin, vimentin, and Slug and normalized with $\beta$-actin in bladder cancer 5637 and T24 cell lines. (B) 5637 and T24 cells treated with various doses of tetrandrine were subjected to western blotting for E-cadherin (E-Ca), N-cadherin (E-Ca), vimentin, Slug and $\beta$-actin. Representative blots from three experiments are shown. (C) F-actin staining with immunofluorescence microscopy was carried out to detect the mobility capacity of 5637 cells treated with tetrandrine.

which indicated that tetrandrine had low cytotoxity on normal cells. While the growth of bladder cancer 5637 and T24 cells was significantly inhibited by tetrandrine at a concentration of $\geq 0.5 \mu \mathrm{M}$, and at cell density $>90 \%$ (Fig. 1C-F). Based on 

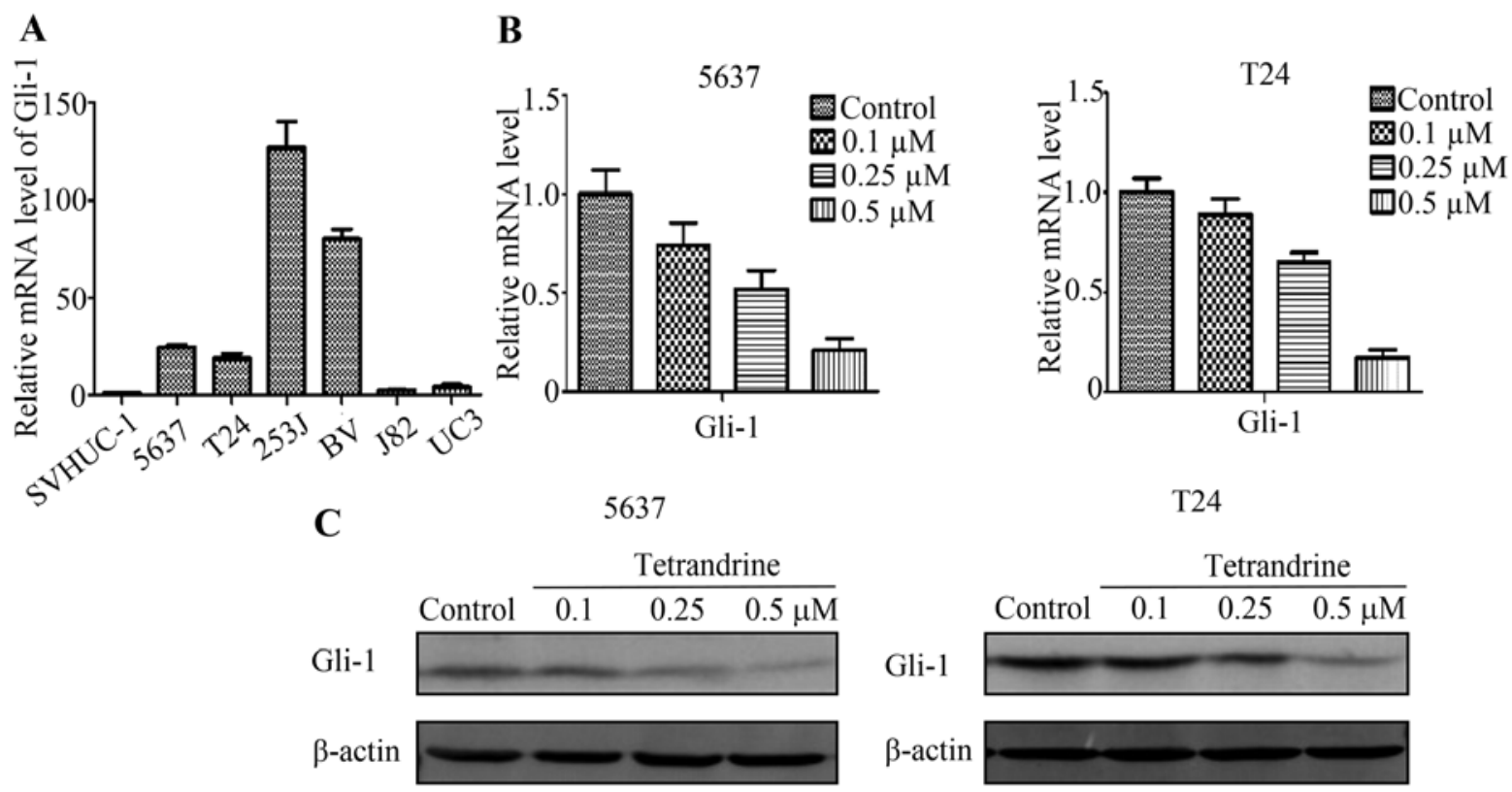

Figure 4. Tetrandrine decreased the expression of Gli-1 in bladder cancer cells. (A) Quantitative real-time PCR was performed to examine the expression of Gli-1 in normal bladder epithelial SVHUC-1 cells and 6 bladder cancer cells. Additionally, the Gli-1 level in bladder cancer 5637 and T24 cells treated with tetrandrine, or not, was detected through quantitative real-time PCR (B) and western blotting (C). Quantification of three independent experiments is shown with error bars representing SEM.

the above, tetrandrine at $0.5 \mu \mathrm{M}$ (a $<10 \%$ inhibitory rate) was chosen as the appropriate dose in the subsequent studies, to exclude the suppressing interference from bladder cancer proliferation by tetrandrine.

Tetrandrine suppresses the migration and invasion of bladder cancer 5637 and T24 cells. In order to determine the function of tetrandrine on bladder cancer cell migration, wound healing assay and Transwell migration assay were assessed under a microscope at 0 and $24 \mathrm{~h}$. The results showed that the scratches of the cells treated with tetrandrine were much wider than that in control group (Fig. 2A and B). Furthermore, tetrandrine in the 5637 cells decreased cell migratory ability after $24 \mathrm{~h}$ (Fig. 2C). Similarly, treatment of the T24 cells with tetrandrine resulted in a prominent decrease in migration compared to the control group (Fig. 2D). These results indicated that tetrandrine was able to repress migration in the human bladder cancer 5637 and T24 cells. Next, using a Matrigel invasion assay, we focused on whether tetrandrine could affect the invasiveness of bladder cancer cells. The results showed that tetrandrine significantly decreased invasive capability of 5637 and T24 cells (Fig. 2C and D).

These results indicated that tetrandrine might play a crucial role in inhibiting the migration and invasion potential of human bladder cancer cells, as presented by the observation in the wound-healing and Transwell assays.

Tetrandrine reverses epithelial-mesenchymal transition (EMT) in human bladder cancer cells. EMT is essential in tumorigenesis and cancer progression (24). We, therefore, examined the effect of tetrandrine on the mRNA and protein expression of EMT markers in bladder cancer cells. The results showed that the expression of $\mathrm{N}$-cadherin, vimentin and Slug reduced, while the expression of E-cadherin increased in a concentration-dependent manner (Fig. 3A and B), suggesting that tetrandrine could block EMT. Then we detected the stress fibers (F-actin) in bladder cancer 5637 cells treated with tetrandrine, or not, with confocal immunofluorescence microscopy. Interestingly, we found that in tetrandrine-treated cells, the bundled F-actin (stress fibers) drastically decreased (Fig. 3C), which indicated the mobility of 5637 cells was inhibited by tetrandrine.

Tetrandrine downregulates the expression of Gli-1 in bladder cancer 5637 and T24 cells. Hedgehog (Hh) signaling pathway has been reported to play important roles in tumorigenesis and progression of several human cancers (25-27). Gli-1 is a key member of Hh signaling pathway signaling (28). Therefore, we detected Gli-1 level in normal bladder epithelial cells and 6 bladder cancer cell lines (5637, T24, 253, BV, J82, UC3). Total RNA was extracted from these cell lines, and through quantitative real-time PCR analysis, we found that expression of Gli-1 in normal bladder epithelial cells is significantly lower than that in bladder cancer cells (Fig. 4A), which indicated that the relative level of Gli-1 is upregulated in bladder cancer cells.

To confirm the ability of tetrandrine to inhibit the $\mathrm{Hh}$ signaling pathway, 5637 and T24 cells treated with tetrandrine for $24 \mathrm{~h}$ were used to examine changes in the Gli-1 level. As shown in Fig. 4B and C, tetrandrine downregulated Gli-1 expression in a dose-dependent manner in mRNA and protein levels. These observations indicated that Hh signaling pathway may play an important role in the pathogenesis of bladder cancer.

Tetrandrine inhibits migration and invasion of bladder cancer cells at least partly through downregulating Gli-1. To determine whether tetrandrine negatively regulate Gli-1 to modulate tumor migration and invasion of bladder cancer, we 


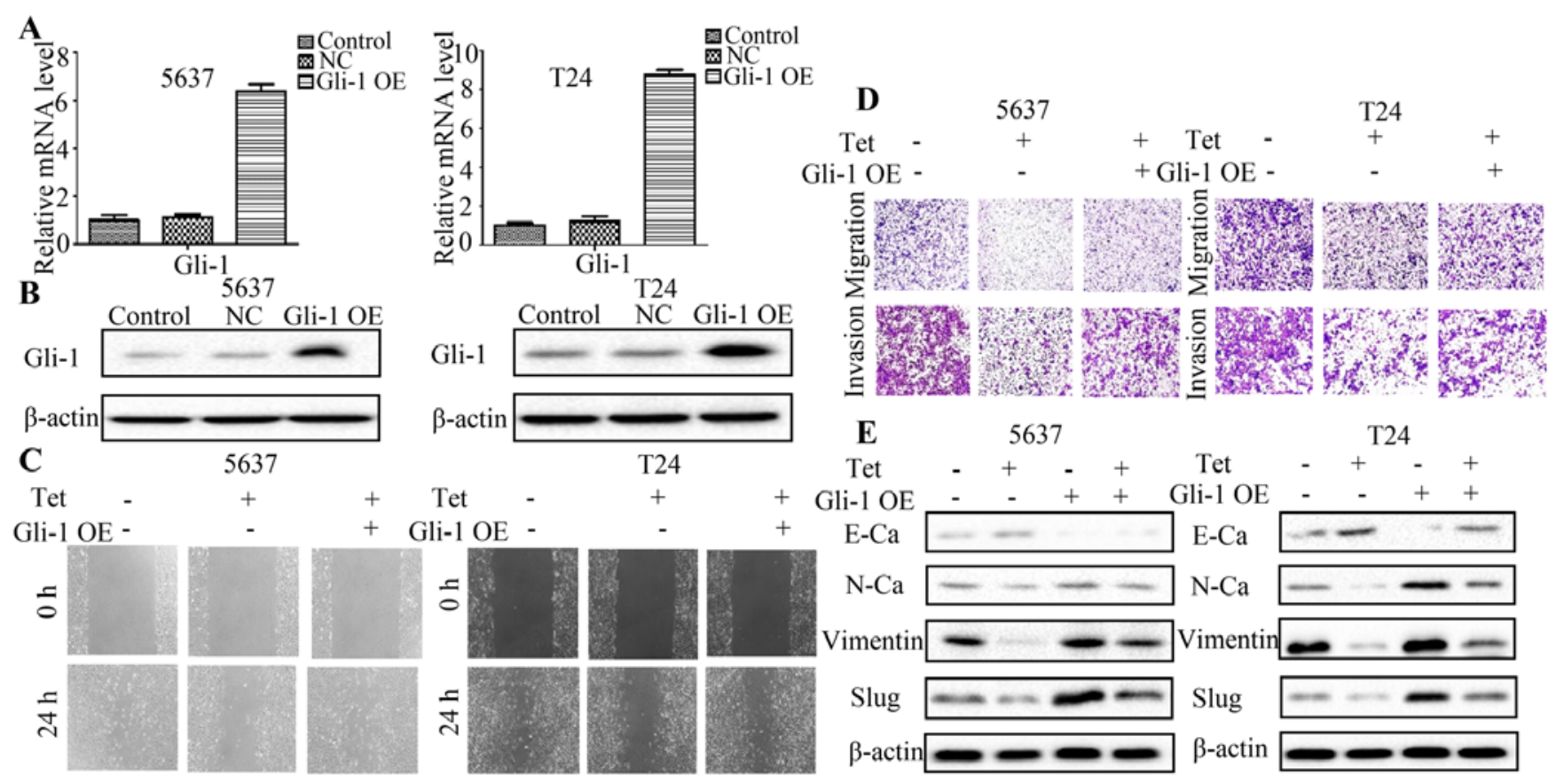

Figure 5. Overexpression (OE) of Gli-1 attenuates the antitumor effect of tetrandrine (tet) in bladder cancer cells. Quantitative real-time PCR (A) and western blotting (B) were performed to detect the Gli-1 level in control, NC (negative control) and Gli-1 OE (Gli-1 overexpression) group. (C) Using wound healing assay, the width of scratches was detected in three different group. (D) Transwell migration assay and Matrigel invasion assay were performed on 5637 and T24 cells with different treatments. Five fields were randomly chosen and visualized by microscopy to evaluate the ability of cell migration and invasiveness. Quantification of three independent experiments is shown, with error bars representing SEM. (E) Western blotting was used to detect the levels of E-cadherin $(\mathrm{E}-\mathrm{Ca}), \mathrm{N}$-cadherin $(\mathrm{N}-\mathrm{Ca})$, vimentin, Slug and $\beta$-actin protein with various treatments in 5637 and T24 cells. Representative blots of three experiments are shown.

overexpressed Gli-1 by transiently transfecting Gli-1 plasmid into 5637 and T24 cells (Fig. 5A and B). Tetrandrine decreased the width of scratches and reduced the cell number in the lower transwell chamber, while combined Gli-1 overexpression potentiated the migration and invasion ability of 5637 and T24 cells (Fig. 5C and D). These results indicated that Gli-1 participated in the antitumor effect of tetrandrine on migration and invasion in bladder cancer. Through western blot analysis of EMT markers, we further found that the elevated level of E-cadherin and the reduced expression of $\mathrm{N}$-cadherin, vimentin and Slug by tetrandrine could be partially reversed when combined with the Gli-1 overexpression in bladder cancer cells (Fig. 5E). Taken together, these results suggest a deeper concept that tetrandrine might inhibit bladder cancer migration and invasion, and reverse EMT at least partly through negatively regulating Gli-1.

\section{Discussion}

Numerous studies in the literature have shown that tetrandrine exhibits strong antitumor effect against various cancer. However, there have been only a limited number of studies on the anti-metastasis activity of tetrandrine on cancer cells. It has been reported that tetrandrine significantly inhibited tumor metastasis in a mouse model of stage IV breast cancer, involving the endothelial cell-specific molecule-1 (ESM-1), integrin $\beta 5$ protein and intercellular cell adhesion molecule-1 (ICAM-1). In our earlier study (data not shown), we found that tetrandrine could inhibit metastatic phenotype of prostate cancer cells by regulating AKT/mTOR/MMP-9 signaling pathway. In this study, our results demonstrated that tetrandrine significantly inhibited bladder cancer migration and invasion, suggesting that tetrandrine might be a potential therapeutic method for bladder cancer.

Epithelial-mesenchymal transition (EMT) is a process in which epithelial cells lose their characteristics and gain mesenchymal features. EMT could result in invasion of surrounding stroma, intravasation, dissemination and colonization of distant sites. Therefore, EMT may confer metastatic characteristics on carcinomas to some extent. Studies have shown that EMT has a positive impact on HCC invasion and metastasis (29). A hallmark of EMT is the downregulation of E-cadherin (30). E-cadherin is a cell adhesion molecule involved in the cell-cell adhesion. The decreased level of E-cadherin is considered to indicate elevated metastatic activity of cancer. While the increased expression of $\mathrm{N}$-cadherin, vimentin and Slug are correlated with poor prognosis and tumor aggressiveness $(31,32)$. In our study, we observed an induced expression of E-cadherin while a reduced expression of N-cadherin, vimentin, Slug and a decreased mobility in bladder cancer cells treated with tetrandrine, suggesting a potential role of tetrandrine in the blockade of EMT involved in early metastasis.

Hedgehog (Hh) pathway has been verified to play a momentous role in embryonic development and the tumor progression. Previous studies have shown that the Gli-1 is a vital effector of the Hedgehog pathway (33). High expression of Gli-1 has a positive correlation with the invasiveness of 
breast cancer (34), and with the poor prognosis of disease (35). Additionally, we found that the mRNA level of Gli-1 in 6 different bladder cancer cells is significantly higher than that in bladder epithelial SVHUC-1 cells. Studies indicated that aberrant activation of Hedgehog $(\mathrm{Hh})$ could facilitate cancer EMT, invasion, and metastasis (36). In gastric cancer, Hh signaling pathway potentiates metastasis by positively regulating Akt, EMT and MMP-9 pathway (37). Additionally, it was reported that hypoxia can upregulate Gli-1 to induce EMT and to enhance tumor invasiveness in pancreatic cancer (38). Accumulating evidence indicates that Hedgehog signaling pathway especially Gli-1 is correlated with EMT, and tumor invasion and metastasis. However, the roles of Gli-1 in the tetrandrine-induced EMT and invasion of bladder cancer cells have not been clarified. Our findings show that tetrandrine combined with Gli-1 overexpression could attenuate the anti-metastatic capability of tetrandrine on bladder cancer. Furthermore, the expression of E-cadherin was down while the N-cadherin, vimentin and Slug levels recovered to some extent. These findings deepen the concept that Hedgehog signaling is a fundamental driver of tumor metastasis in bladder cancer, thus Hedgehog pathway blockage by tetrandrine is a promising novel treatment strategy for unresectable forms of bladder cancer.

Taken together, our above results demonstrated that tetrandrine inhibits bladder cancer cell migration and invasion, and reverses EMT in vitro by negatively regulating Gli-1. It is suggested that Gli-1 could be potential therapeutic target of tetrandrine against bladder cancer.

\section{Acknowledgements}

This study was partially supported by the Institutional Scientific Development Foundation of the First Affiliated Hospital of Xi'an Jiaotong University.

\section{References}

1. Siegel RL, Miller KD and Jemal A: Cancer statistics, 2015. CA Cancer J Clin 65: 5-29, 2015

2. Sylvester RJ, van der Meijden AP, Oosterlinck W, Witjes JA, Bouffioux C, Denis L, Newling DW and Kurth K: Predicting recurrence and progression in individual patients with stage Ta T1 bladder cancer using EORTC risk tables: a combined analysis of 2596 patients from seven EORTC trials. Eur Urol 49: 465-466, discussion 475-477, 2006

3. Nisticò P, Bissell MJ and Radisky DC: Epithelial-mesenchymal transition: General principles and pathological relevance with special emphasis on the role of matrix metalloproteinases. Cold Spring Harb Perspect Biol 4: 4, 2012.

4. Chang CJ, Chao CH, Xia W, Yang JY, Xiong Y, Li CW, Yu WH, Rehman SK, Hsu JL, Lee HH, et al: p53 regulates epithelialmesenchymal transition and stem cell properties through modulating miRNAs. Nat Cell Biol 13: 317-323, 2011.

5. Xie D, Gore C, Liu J, Pong RC, Mason R, Hao G, Long M, Kabbani W, Yu L, Zhang H, et al: Role of DAB2IP in modulating epithelial-to-mesenchymal transition and prostate cancer metastasis. Proc Natl Acad Sci USA 107: 2485-2490, 2010.

6. Gjerdrum C, Tiron C, Høiby T, Stefansson I, Haugen H, Sandal T, Collett K, Li S, McCormack E, Gjertsen BT, et al: Axl is an essential epithelial-to-mesenchymal transition-induced regulator of breast cancer metastasis and patient survival. Proc Natl Acad Sci USA 107: 1124-1129, 2010.

7. Wang H, Li YY, Wu YY and Nie YQ: Expression and clinical significance of hedgehog signaling pathway related components in colorectal cancer. Asian Pac J Cancer Prev 13: 2319-2324, 2012.
8. Flemban A and Qualtrough D: The potential role of hedgehog signaling in the luminal/basal phenotype of breast epithelia and in breast cancer invasion and metastasis. Cancers (Basel) 7: $1863-1884,2015$

9. Lin H, Jackson GA, Lu Y, Drenkhahn SK, Brownstein KJ, Starkey NJ, Lamberson WR, Fritsche KL, Mossine VV, BeschWilliford CL, et al: Inhibition of Gli/hedgehog signaling in prostate cancer cells by "cancer bush" Sutherlandia frutescens extract. Cell Biol Int 40: 131-142, 2016.

10. Huang L, Walter V, Hayes DN and Onaitis M: Hedgehog-GLI signaling inhibition suppresses tumor growth in squamous lung cancer. Clin Cancer Res 20: 1566-1575, 2014.

11. Li X, Deng W, Nail CD, Bailey SK, Kraus MH, Ruppert JM and Lobo-Ruppert SM: Snail induction is an early response to Gli1 that determines the efficiency of epithelial transformation. Oncogene 25: 609-621, 2006.

12. Chen JS, Huang XH, Wang Q, Huang JQ, Zhang LJ, Chen XL, Lei $\mathbf{J}$ and Cheng ZX: Sonic hedgehog signaling pathway induces cell migration and invasion through focal adhesion kinase/ AKT signaling-mediated activation of matrix metalloproteinase (MMP)-2 and MMP-9 in liver cancer. Carcinogenesis 34: 10-19, 2013.

13. Chaudhary P and Vishwanatha JK: c-Jun NH2-terminal kinaseinduced proteasomal degradation of c-FLIPL/S and $\mathrm{Bcl} 2$ sensitize prostate cancer cells to Fas- and mitochondria-mediated apoptosis by tetrandrine. Biochem Pharmacol 91: 457-473, 2014.

14. Liu W, Kou B, Ma ZK, Tang XS, Lv C, Ye M, Chen JQ, Li L, Wang XY and He DL: Tetrandrine suppresses proliferation, induces apoptosis, and inhibits migration and invasion in human prostate cancer cells. Asian J Androl 17: 850-853, 2015.

15. Wu K, Zhou M, Wu QX, Yuan SX, Wang DX, Jin JL, Huang J, Yang JQ, Sun WJ, Wan LH, et al: The role of IGFBP-5 in mediating the anti-proliferation effect of tetrandrine in human colon cancer cells. Int J Oncol 46: 1205-1213, 2015.

16. Wu JM, Chen Y, Chen JC, Lin TY and Tseng SH: Tetrandrine induces apoptosis and growth suppression of colon cancer cells in mice. Cancer Lett 287: 187-195, 2010.

17. Qin R, Shen H, Cao Y, Fang Y, Li H, Chen Q and Xu W: Tetrandrine induces mitochondria-mediated apoptosis in human gastric cancer BGC-823 cells. PLoS One 8: e76486, 2013.

18. Wang TH, Wan JY, Gong X, Li HZ and Cheng Y: Tetrandrine enhances cytotoxicity of cisplatin in human drug-resistant esophageal squamous carcinoma cells by inhibition of multidrug resistance-associated protein 1. Oncol Rep 28: 1681-1686, 2012.

19. Sun YF and Wink M: Tetrandrine and fangchinoline, bisbenzylisoquinoline alkaloids from Stephania tetrandra can reverse multidrug resistance by inhibiting P-glycoprotein activity in multidrug resistant human cancer cells. Phytomedicine 21: 1110-1119, 2014

20. Gao JL, Ji X, He TC, Zhang Q, He K, Zhao Y, Chen SH and Lv GY: Tetrandrine suppresses cancer angiogenesis and metastasis in 4T1 tumor bearing mice. Evid Based Complement Alternat Med 2013: 265061, 2013.

21. Xiao W, Jiang Y, Men Q, Yuan L, Huang Z, Liu T, Li W and Liu X: Tetrandrine induces G1/S cell cycle arrest through the ROS/Akt pathway in EOMA cells and inhibits angiogenesis in vivo. Int J Oncol 46: 360-368, 2015.

22. He BC, Gao JL, Zhang BQ, Luo Q, Shi Q, Kim SH, Huang E, Gao Y, Yang K, Wagner ER, et al: Tetrandrine inhibits Wnt/ $\beta$-catenin signaling and suppresses tumor growth of human colorectal cancer. Mol Pharmacol 79: 211-219, 2011.

23. Li X, Su B, Liu R, Wu D and He D: Tetrandrine induces apoptosis and triggers caspase cascade in human bladder cancer cells. J Surg Res 166: e45-e51, 2011.

24. Iwatsuki M, Mimori K, Yokobori T, Ishi H, Beppu T, Nakamori S, Baba $\mathrm{H}$ and Mori M: Epithelial-mesenchymal transition in cancer development and its clinical significance. Cancer Sci 101: 293-299, 2010.

25. Amakye D, Jagani $Z$ and Dorsch $M$ : Unraveling the therapeutic potential of the Hedgehog pathway in cancer. Nat Med 19: $1410-1422,2013$

26. Briscoe $J$ and Thérond PP: The mechanisms of Hedgehog signalling and its roles in development and disease. Nat Rev Mol Cell Biol 14: 416-429, 2013.

27. Harris LG, Pannell LK, Singh S, Samant RS and Shevde LA: Increased vascularity and spontaneous metastasis of breast cancer by hedgehog signaling mediated upregulation of cyr61. Oncogene 31: 3370-3380, 2012 . 
28. Zhang J, Zhang GX, Chen FF, He BS, Ye F and Pan XL: Effect of Helicobacter pylori lipopolysaccharide on expression of Gli and Ptch-1 proteins in sonic hedgehog signaling pathway of gastric mucosa GES-1 cells. Zhejiang Da Xue Xue Bao Yi Xue Ban 42: 543-549, 2013. (In Chinese).

29. Reichl P, Haider C, Grubinger M and Mikulits W: TGF- $\beta$ in epithelial to mesenchymal transition and metastasis of liver carcinoma. Curr Pharm Des 18: 4135-4147, 2012.

30. Mallini P, Lennard T, Kirby J and Meeson A: Epithelial-tomesenchymal transition: What is the impact on breast cancer stem cells and drug resistance. Cancer Treat Rev 40: 341-348, 2014.

31. Satelli A and Li S: Vimentin in cancer and its potential as a molecular target for cancer therapy. Cell Mol Life Sci 68: 3033-3046, 2011.

32. Voulgari A and Pintzas A: Epithelial-mesenchymal transition in cancer metastasis: Mechanisms, markers and strategies to overcome drug resistance in the clinic. Biochim Biophys Acta 1796: 75-90, 2009.

33. Li X, Ma Q, Duan W, Liu H, Xu H and Wu E: Paracrine sonic hedgehog signaling derived from tumor epithelial cells: A key regulator in the pancreatic tumor microenvironment. Crit Rev Eukaryot Gene Expr 22: 97-108, 2012.
34. Jeng KS, Sheen IS, Jeng WJ, Yu MC, Hsiau HI and Chang FY: High expression of Sonic Hedgehog signaling pathway genes indicates a risk of recurrence of breast carcinoma. Onco Targets Ther 7: 79-86, 2013.

35. ten Haaf A, Bektas N, von Serenyi S, Losen I, Arweiler EC, Hartmann A, Knüchel R and Dahl E: Expression of the gliomaassociated oncogene homolog (GLI) 1 in human breast cancer is associated with unfavourable overall survival. BMC Cancer 9: 298, 2009.

36. Abidi A: Hedgehog signaling pathway: a novel target for cancer therapy: vismodegib, a promising therapeutic option in treatment of basal cell carcinomas. Indian J Pharmacol 46: 3-12, 2014.

37. Yoo YA, Kang MH, Lee HJ, Kim BH, Park JK, Kim HK, Kim JS and Oh SC: Sonic hedgehog pathway promotes metastasis and lymphangiogenesis via activation of Akt, EMT, and MMP-9 pathway in gastric cancer. Cancer Res 71: 7061-7070, 2011.

38. Lei J, Ma J, Ma Q, Li X, Liu H, Xu Q, Duan W, Sun Q, Xu J, Wu Z, et al: Hedgehog signaling regulates hypoxia induced epithelial to mesenchymal transition and invasion in pancreatic cancer cells via a ligand-independent manner. Mol Cancer 12: 66, 2013. 